\title{
Evaluación psicológica de la comunicación relacional no verbal
}

Rozzana Sánchez-Aragón*

* Doctora en Psicología Social. Profesora titular de la Facultad de Psicología, Universidad Nacional Autónoma de México, México, D.F., México.

Correo electrónico:

rozzara@unam.mx

Recibido: 25 de abril del 2014

Aprobado: 3 de julio del 2014

Cómo citar este artículo: Sánchez-Aragón, R. (2014). Evaluación psicológica de la comunicación relacional no verbal. Pensando Psicología, 10(17), 27-42. doi: http://dx.doi. org/10.16925/pe.v10i17.782

\section{Resumen}

La comunicación relacional representa el principal mecanismo de interacción entre los miembros de una relación cercana, por lo que en sus dos vías (verbal y no-verbal) contribuye a desarrollar un vínculo ya sea de intimidad o de hostilidad entre sus miembros. La comunicación no verbal, por su carácter complejo, no ha sido fácil o frecuentemente operacionalizada (p. e., Burgoon y Hale, 1987; Kahn, 1970; Riggio, 2006) y aún menos en el contexto de la cultura mexicana, la cual se caracteriza por el gran peso que tienen las relaciones personales (Díaz, 1994). Por lo anterior, el propósito del presente estudio fue desarrollar y validar dos autorreportes desde la visión del participante con respecto a los mensajes recibidos a partir del comportamiento no verbal de su pareja. Para ello, se trabajó con una muestra no probabilística de 367 adultos de la Ciudad de México con pareja, quienes respondieron a la "Escala de comunicación no verbal - percepción" y la "Escala de comunicación no verbalinterpretación". Los resultados mostraron dos medidas válidas y confiables cuyos factores se apoyan fuertemente en la literatura sobre relaciones personales y comunicación.

Palabras clave: comunicación, interacción, no verbal, medición, pareja.

\section{Psychological Evaluation of Non-Verbal Relational Communication}

\section{Abstract}

Relational communication represents the primary interaction mechanism between members of a close relationship, as it plays a role in developing bonds-either of intimacy or hostility-between the people in the relationship across both communication channels (verbal and non-verbal). Due to its complex nature, non-verbal communication has not been easily or frequently operationalized (for example, Burgoon \& Hale, 1987; Kahn, 1970; Riggio, 2006), and even less so in the context of the Mexican culture, which is characterized by the great importance of personal relationships (Díaz, 1994). Thus, the aim of this study is to develop and validate two self-reports on participants' perspectives of messages received based on their partners' non-verbal behavior. The study used a non-probability sample of 367 adults with partners in Mexico City, who responded to the "Non-Verbal Communication Scale - Perception" and the "Non-Verbal Communication Scale - Interpretation" self-reports. The results showed two valid and reliable measures whose factors were strongly supported by the literature on personal relationships and communication.

Keywords: communication, interaction, non-verbal, measurement, partner.

\section{Avaliação psicológica da comunicação relacional não verbal}

\section{Resumo}

A comunicação relacional representa o principal mecanismo de interação entre os membros de uma relação próxima, razão pela qual em suas duas vias (verbal e não verbal) contribui para o desenvolvimento de um vínculo, seja de intimidade ou de hostilidade entre seus membros. A comunicação não verbal, por seu caráter complexo, não tem sido fácil ou frequentemente operacionalizada (p. e., Burgoon e Hale, 1987; Kahn, 1970; Riggio, 2006) e ainda menos no contexto da cultura mexicana, a qual se caracteriza pelo grande peso que as relações pessoais têm (Díaz, 1994). Portanto, o propósito do presente estudo foi desenvolver e validar dois "autorrelatórios" a partir da visão do participante a respeito das mensagens recebidas a partir do comportamento não verbal de seu/sua companheiro/a. Para isso, trabalhou-se com uma amostra não probabilística de 367 adultos da Cidade do México com casal, os quais responderam à "Escala de comunicação não verbal - percepção" e à "Escala de comunicação não verbal - interpretação". Os resultados mostraram duas medidas válidas e confiáveis, cujos fatores se apoiam fortemente na literatura sobre relações pessoais e comunicação.

Palavras-chave: comunicação, interação, não verbal, medição, casal. 


\section{Introducción $^{1}$}

La capacidad de comunicarse es vital para la supervivencia de las especies animales, algunas de las cuales tienen impresionantes formas de transmitirse información, aunque ninguna alcanza la precisión y la flexibilidad que caracteriza la comunicación humana (Deacon, 1997; Hauser, 1996), pues esta requiere una coordinación entre los interlocutores que se observa en la forma de regular sus palabras, sonidos y movimientos, facilitando con ello la atracción mutua y la comprensión compartida con otros (Giles, Coupland y Coupland, 1991).

El proceso de comunicación supone el uso de canales para la transmisión del mensaje y de códigos que llevan el mensaje; ante la ausencia de alguno de estos dos elementos, simplemente no hay comunicación. Un canal implica un cierto aparato físico que transmite mensajes codificados de una manera determinada, como, por ejemplo, la voz y el oído humano, ya que los mensajes fluyen a través de estos aparatos y se suelen codificar por medio de las palabras (verbal) y por medio de señales no verbales como suspiros, gruñidos, risa, gritos y movimientos, entre otros (Danziger, 1982).

\section{Tipos de comunicación}

Todas las formas de comunicación se pueden clasificar en verbales y no verbales, y estas a su vez se pueden subdividir en vocales y no vocales. Gran parte de la comunicación que tiene lugar entre las personas es verbal vocal, es decir, que se basa en el lenguaje hablado o la palabra, y emplea signos orales y hablados (Cortés, 2010). La comunicación verbal no vocal incluye, tanto la comunicación por escrito, como la comunicación que se transmite a través del lenguaje de señas, deletreo con los dedos, Braille y demás alternativas del lenguaje verbal.

La comunicación no verbal vocal puede ser vocal cuando se enfoca en las características vocales como, por ejemplo, el tono, la velocidad y la modulación, y puede ser no verbal no vocal cuando se centra en el lenguaje corporal, el medio ambiente, la vestimenta, la conducta espacial, el tacto, la conducta visual, las expresiones faciales, los movimientos y las posturas corporales, entre otras (Kleinke, 1986).

Es importante mencionar que todo tipo de comu-

Agradecimiento a la Dirección General de Apoyo al Personal Académico de la UNAM por su financiamiento al Proyecto PAPIIT IN 302811-3, "Del Individuo a la pareja: el papel de las emociones, la comunicación la atribución y la regulación emocional”. nicación tiene como objetivo expresar poder, estatus, influencia o atracción, así como la obtención de información (Kimble et al. 2002). En este mismo sentido, todo acto de comunicar lleva un "mandato" por lo menos implícito, de entrar en algún tipo de relación con el que se está comunicando (Floyd y Burgoon, 1999) y hace uso del intercambio de información sobre un tema particular a la vez que de formas de imposición conductual en cada acto de comunicación (Ruesch y Bateson, 1951).

Unidos al carácter verbal y vocal o no de la comunicación, existen dos elementos que determinan conjuntamente la interacción, la afectividad y el conocimiento que se genera en una relación, y que forman parte del proceso comunicativo; estos son el contenido -que comprende los temas o las áreas de interés sobre los cuales una diada se comunica, su frecuencia o cantidad- y el estilo de comunicación - que refleja la(s) manera(s) como esa información es transmitida (Sánchez-Aragón y Díaz-Loving, 2003)-.

En lo que respecta al contenido de la comunicación en las relaciones de pareja o románticas, Noller y Fitzpatrick (1990) reportaron que las parejas satisfechas manifestaron ocupar más tiempo hablando y discutiendo temas personales y menos tiempo en conflictos, en comparación con las parejas insatisfechas. En otras investigaciones previas a los noventa sobre los temas de la comunicación, se encontró que en este contexto interpersonal es importante hablar de áreas como la solución de problemas (Miller, Nunnally y Wackman, 1976; Bernhard, 1991); la familia, la vida sexual y las actitudes (Shapiro y Swensen, 1977; Pick de Weiss, 1986); los sentimientos o afectos (Bernhard, 1991; Davidson, Balswick y Halverson, 1983; Levinger y Senn, 1967; O’Neill y O’Neill, 1974; Satir, 1986); las ansiedades (Schumm, Figley y Jurich, 1979); el trabajo o la escuela, el mantenimiento del hogar, otras relaciones, conversaciones con otros y comida (Dickson, Markman y Markman, 1988; citados en Noller y Fitzpatrick, 1990).

Posteriormente, en México, Estrella (1991) sugiere que los temas que se abordan se pueden dividir en doce áreas:

- Afecto: información que da un cónyuge a otro respecto a sentimientos negativos o positivos hacia su pareja.

- Emoción: información que expresa el estado de ánimo hacia su pareja o hacia sí mismo.

- Familia extendida: información que se da sobre la familia del cónyuge o sobre su propia familia. 
- Vida sexual: información sobre aspectos de la vida sexual de la pareja.

- Amistades: información sobre sus relaciones con amistades propias y de su pareja.

- Hijos: información que se refiere a aspectos relacionados con los hijos.

- Vida laboral: información referente al trabajo fuera del hogar que cada cónyuge desempeña.

- Relación marital: información sobre creencias, actitudes y opiniones respecto de la relación de pareja.

- Vida diaria: información que se expresa sobre eventos cotidianos, sociales y temas superfluos.

- Expectativas: opiniones o pensamientos sobre su futuro con su pareja.

- Economía y tiempo libre: información que se expresa sobre la distribución del dinero y la recreación compartida.

- Atracción: información sobre el conjunto de cualidades que le agradan y hacen deseable al cónyuge.

Esta misma autora refiere que las parejas que llevan menos de 5 años de casados hablan más sobre la relación marital, la vida sexual, el trabajo, los sentimientos, las emociones y los disgustos, mientras que las parejas que llevan entre 6 y 10 años de matrimonio platican más sobre los hijos, quizá porque en esta etapa tienen niños pequeños y la comunicación sobre ellos es necesaria (Estrella, 1991).

En cuanto a los estilos de comunicación identificados en México, sobresalen dos estudios: el primero realizado por Estrella (1991) -en el cual encuentra cuatro estilos de comunicación claramente diferenciados: positivo, reservado, violento y negativo- y el segundo, de Sánchez-Aragón y Díaz-Loving (2003), los cuales identifican dos estilos diferenciales: cuando la persona se describe a sí misma y cuando describe a su pareja en términos de estilos de comunicación. Las formas observadas fueron llamadas: social afiliativo, social automodificador, simpático, abierto, social normativo, reflexivo, claro, violencia instrumental, evitante, hiriente expresivo, autoritario e irritante expresivo, las cuales caracterizan más claramente a las parejas mexicanas cuando se comunican mutuamente.

No obstante, la importancia de estas características del proceso de comunicación relacional (Guerrero, Andersen y Afifi, 2007), la vía no verbal ha sido destacada, al afirmar que sólo el 7\% se debe al contexto expresado por la palabra hablada o escrita, mientras que el 55\% de la totalidad de este impacto está relacionada con la expresión del rostro y el $38 \%$ restante a la voz del comunicante (Mehrabian, 1968), por lo que a continuación se abordará más específicamente.

\section{Comunicación no verbal}

Para empezar basta con citar a Watzlawick (1974), quien señala que es imposible no comunicar, ya que todo lo que se diga (o no) o se haga (o no), va a transmitir un mensaje a la otra persona, y es precisamente por eso que la comunicación no verbal es una parte esencial e inherente en la creación, producción e interpretación del mensaje (Burgoon, 1994; Giles y Le Poire, 2006).

Keeley y Hart (1994) indican que la comunicación no verbal son los signos y símbolos que pueden dar un mensaje, basados en la interpretación y el uso constante, además de que contribuye más a la experiencia íntima de las personas que la comunicación verbal.

Andersen (1999) menciona que este tipo de comunicaciones es multimodal, es decir, que mientras las personas pueden decir una sola palabra a la vez, pueden emplear numerosas señales no verbales que puedan enviar más mensajes, atraer la atención de la otra persona y generar con ello mayor grado de intimidad, ya que son vistas como una totalidad. Asimismo, indica que muchas de las conductas no verbales son espontáneas y ejercen gran poder ya que son percibidas como auténticas (Andersen, Guerrero y Jones, 2006).

Cestero (2006) conceptualiza la comunicación no verbal como todos los signos y sistemas de signos no lingüísticos que comunican o se utilizan para comunicar, incluyendo hábitos y costumbres culturales.

Por su parte, Manusov y Patterson (2006) la definen como un proceso que comprende el envío y la recepción de información por medio de la apariencia, los objetos y el ambiente, además de la conducta en diversos escenarios sociales.

Con base en estas definiciones, es posible identificar las diferentes vías de comunicación no verbal que forman parte de lo que se ha llamado "análisis de la conversación" y que involucran -desde la perspectiva de Cestero (2000) - los llamados sistemas de comunicación no verbal que se describen en seguida.

\section{Sistemas de comunicación no verbal}

\section{Distancia personal o proxémica}

Se refiere al estudio sistemático de las características espaciales entre una persona y otra durante la interacción 
social (Hall, citado por Danziger, 1982). Cuando se habla con una persona, la distancia ordinaria es de 120 y $150 \mathrm{~cm}$, y si alguno de los dos se acercara o se alejara, esta situación generaría - de inicio- sentimientos de incomodidad. No obstante, cuando se trata de una relación íntima o cercana, de 0 a $45 \mathrm{~cm}$ se siente apropiado. Esto indica que las distancias interpersonales aceptables varían con base en el nivel de cercanía entre las personas (Coker y Burgoon, 1987), como es el caso de los amigos, las pareja y las personas que les agradan (Sundstorm y Alman, 1976), pues la proximidad permite el paso de la comunicación de un nivel superficial a uno más profundo, involucrando aspectos como el afecto (Floyd y Morman, 1997), entre otros.

\section{Contacto visual}

Es una característica del modo de mirar que ocurre cuando dos personas se observan fija y directamente a los ojos durante la conversación por un largo periodo, mientras que, en otros momentos, se ve a la otra persona sin que esta corresponda de la misma manera e incluso evite la mirada mutua debido a cierta vergüenza o confusión causada por la "invasión de la privacidad". Cuando hay un nivel elevado de contacto visual recíproco es porque hay una gran tolerancia al acercamiento o la intimidad mutua (Danziger, 1982).

\section{Expresiones faciales}

El rostro es considerado la fuente más importante y confiable de información acerca de las emociones de una persona, además de ser un área corporal capaz de asumir diversos aspectos en un lapso corto. La cara es la única parte del cuerpo que tiene músculos que no sirven para moverse sino para "mover a otros" (Smith y Scott, citado en Ruys y Stapel, 2008), ya que es capaz de generar diferentes estados emocionales y comunica aquellos que vive el emisor de la información. Dentro de este mismo rubro de la expresión facial, pero en torno a la sonrisa, Palmer y Simmons (1995) reportaron que el incremento del agrado y la intimidad por parte del receptor se ve reflejado en un aumento en la frecuencia de las sonrisas, ya que: a) una pequeña cantidad de sonrisas se generan en relaciones amistosas; b) una cantidad mediana a grande de sonrisas para conductas de coqueteo (Coker y Burgoon, 1987), y c) sonrisas constantes en interacciones seductoras (Andersen, 1985). Pero es necesario que estas sonrisas sean identificadas como verdaderas o sonrisas Duchenne, que se distinguen porque los labios están plegados hacia arriba y por la aparición de pliegues en los párpados.

\section{Posturas y movimientos corporales}

Estas formas emiten señales claras y dobles que son reconocidas en la medida en que se conoce al emisor. Al respecto, Mehrabian $(1968 ; 1969)$ indica que la cercanía física, un mayor contacto visual, una inclinación hacia adelante y la orientación del torso en dirección de la otra persona, comunican actitudes positivas. Más tarde, este mismo autor (Mehrabian, citado por Kimble et al., 2002) encontró que las posturas de relajación (inclinación del cuerpo hacia atrás o a los lados) son asociadas con un estatus más alto o dominancia, mientras que si las personas mantienen una postura erguida, asimétrica y atenta, se les asocia con un estatus más bajo durante la interacción. Burgoon y Baesler (1991) y Andersen (1999) indican que inclinar el cuerpo hacia delante comunica interés y afecto y se percibe como conducta de intimidad. Así pues, los individuos que se inclinan el uno hacia el otro y que están en el mismo ángulo de orientación son percibidos como más seductores que los que se inclinan en dirección opuesta. Y cuando las parejas se apoyan durante un conflicto, tienden a orientar su cuerpo directamente el uno frente al otro, además de acortar la distancia (Newton y Burgoon, 1990).

\section{Tono de voz}

Para inferir el estado emocional de las personas, aspectos como el volumen, el tono y la velocidad son importantísimos (Planalp, 1998). Un tono de voz agradable es importante ya que ayuda a las personas a iniciar y mantener una relación (Guerrero, 2004). Por tanto, su uso (tono de voz íntimo, menos silencios, más calidez y fluidez) puede caracterizar las relaciones interpersonales (Andersen, 1985). A los aspectos no verbales de la locución se les suele llamar paralenguaje, el cual se refiere a cómo se dice algo y no a qué se dice exactamente. Este acompaña al lenguaje pero no es parte de él (Knapp, 1997).

\section{Contacto físico}

El contacto físico es de vital importancia para el desarrollo del ser humano (Guerrero, 2000). Generalmente 
afecta la experiencia de intimidad en las relaciones interpersonales, tal vez porque esta elimina el espacio que existe entre dos personas (Thayer, 1986). Jones y Yarbrough (1985) identificaron tres tipos de contacto que llevan a las personas a una interacción más íntima: 1) contacto de inclusión, que se da en forma prolongada y transmite o lleva la atención al acto de estar juntos; usualmente interviene la parte baja del cuerpo (piernas, rodillas, caderas o abrazos laterales) y generalmente se da entre parejas o amigos cercanos; 2) contacto sexual, que expresa atracción o un propósito erótico-genital; se dan abrazos y caricias prolongadas que van de una parte del cuerpo a otra, y 3) contacto afectivo, que manifiesta simpatía hacia la otra persona e incluye un amplio rango de conductas tales como poner la mano en el hombro o apretar un brazo. Martson, Hecht, Manke, McDaniel y Reeder (1998) encontraron que las conductas de contacto físico entre las parejas como caricias, besos y abrazos- son la principal forma en que comunican el grado de intimidad en las relaciones románticas.

En este punto cabe indicar que este tipo de comunicación es muy importante en la relación de pareja, ya que la conducta individual de los miembros de la diada va a influir directamente en la conducta del otro (Gable, Reis y Downey, 2003; Shumway y Wampler, 2002). Además, la pareja exige precisión en la expresión de las emociones, pues la mayor parte del tiempo sus integrantes necesitan saber acerca de los sentimientos del otro para así tomarlos en cuenta en la solución de problemas o en la toma de decisiones (Noller, 2006). En este punto, es necesario señalar las contribuciones de Poyatos (2002), que ha identificado en forma muy clara el significado emocional que poseen una serie de conductas no verbales propias de los sistemas de comunicación, según Cestero (2000), lo cual facilita la precisión en las interpretaciones que se dan durante la interacción diádica. Por ejemplo, la sonrisa en conjunto con movimientos de cabeza mostrando inclinación hacia delante, son los indicadores no verbales para la expresión de amor (Gonzaga, Keltner, Londahl y Smith, 2001).

En ese mismo punto, Nowicki y Duke (2001) indicaron así las funciones de este tipo de comunicación: 1) la expresión de las emociones hacia el compañero acerca de otras personas o cosas y 2) revelar mensajes sobre la relación indican tanto el tipo de relación, como su grado de cercanía. Al respecto, Sánchez-Aragón y Díaz-Loving (2003) indican que en la interacción de pareja la comunicación contiene una extensa escala de signos que representan sentimientos e ideas, los cuales permiten a sus miembros usar besos, caricias y comida especial como símbolos de amor; o bien, el silencio y la distancia como muestras de enojo.

Inherente a la comunicación relacional (Guerrero, Andersen, y Afifi, 2007) en su carácter verbal o no verbal, una pieza fundamental es el desarrollo de la habilidad para identificar las expresiones no verbales con exactitud y la capacidad para responder con conductas no verbales en una forma adecuada (Riggio, 2006), es decir, ser capaz de distinguir, interpretar y expresarse mediante conductas no verbales ya que estas definen la relación (Tyler y Feldman, 2005).

Generalmente, las habilidades dentro de la comunicación no verbal dependen de las capacidades de codificar y decodificar las señales que tengan las personas, ya que, a partir de su habilidad, se puede dar una interacción más fluida, precisa, y es entonces cuando los participantes pueden llegar a su objetivo comunicativo. Así, en lo que toca a la decodificación en la comunicación no verbal, la persona requiere principalmente una "sensibilidad no verbal" o empatía y la motivación del observador, para decodificar las expresiones y conductas de las demás personas de una manera exitosa (De Paulo, Rosenthal, Green, y Rosenkrantz, 1982; Trommsdorff y John, 1992). Por otra parte, codificar conductas no verbales involucra la expresión de las experiencias internas de manera que los demás puedan decodificarlo adecuadamente (Burgoon, 1994).

En el contexto de pareja o romántico, autores como Gottman y Porterfield (1981) y Noller (1992) señalan que la exactitud en la decodificación de los mensajes no verbales es más importante para la relación de pareja que codificar los mensajes verbales. En congruencia, Noller (2001) encontró que las personas que tienen una relación feliz son más acertadas en decodificar las conductas no verbales de su pareja que aquellos que no son felices en su relación. De igual forma, las parejas con una relación satisfactoria pueden decodificar con mayor exactitud las conductas de su pareja que las conductas de un desconocido, al contrario de las parejas insatisfechas. Además, notó que la exactitud con que una pareja codifica las conductas no verbales se incrementen a lo largo del tiempo en los tres tipos de mensajes: positivo, negativo y neutral.

Como es evidente, la complejidad inherente a la comunicación no verbal en el contexto de la relación de pareja no sólo ha exigido su definición, mecanismos y reconocimiento de sus consecuencias a partir de la inferencia y algunas investigaciones, sino que también ha sido fundamental el desarrollo de medidas 
psicológicas encaminadas a operacionalizar esta variable tan compleja.

\section{Medición de la comunicación no verbal}

En esta área de conocimiento de la comunicación no verbal se han desarrollado varias medidas cuyo propósito ha sido -en su momento- representar las formas de esta comunicación en diferentes contextos relacionales. Algunas de las contribuciones más representativas son la escala de comunicación marital, la escala de comunicación relacional y el inventario de habilidades sociales, los cuales se describen brevemente en los siguientes apartados.

\section{Escala de comunicación marital (Marital Communication Scale)}

La escala de comunicación marital (Marital Communication Scale) de Kahn (1970) parte de la idea de que las parejas se comunican entre sí por medio de un conjunto estandarizado de mensajes verbales en los que el significado de los mensajes depende cómo se expresan de manera no verbal. Consta de dieciséis ítems e incluye: 1) una breve descripción escrita de una situación hipotética en la que los miembros de la pareja se imaginan a sí mismos, 2) tres posibles mensajes que pueden ser comunicados, dada una situación, y 3) una declaración normalizada que, dependiendo del estilo de la entrega, es capaz de transmitir cualquiera de los mensajes alternativos. En ocho de los ítems, el marido es el remitente y la mujer, la receptora, y en los otros ocho ítems estos papeles se invierten equilibrando el orden. A raíz de la comunicación de cada ítem, emisores y receptores respondieron una pregunta destinada a evaluar su percepción después de haber enviado y recibido el mensaje, respectivamente. Los remitentes respondieron a esta pregunta en una escala de Likert de seis puntos, así: $1=$ "mi comunicación no fue muy clara" y $6=$ "mi comunicación fue muy clara", mientras que a los receptores se les pidió indicar la confianza que tenían en su elección en el mensaje correcto. Esta respuesta también se hizo en una escala de seis puntos, así: 1 = "no confío en que la elección de mi respuesta es correcta" y 6 = "confío plenamente en que la elección de mi respuesta es correcta".

\section{Escala de comunicación relacional (Relational Communication Scale)}

La escala de comunicación relacional (Relational Communication Scale) de Burgoon y Hale (1987) se basa en la concepción de cómo los temas verbales y no verbales presentes en la comunicación de las personas definen una relación interpersonal. Para su diseño, se tuvieron en cuenta doce dimensiones teóricas relacionales de comunicación: dominación-sumisión, intimidad, afecto-hostilidad, intensidad de la participación, inclusiónexclusión, confianza, profundidad-superficialidad, excitación emocional, serenidad, semejanza, formalidad y tareas de orientación social. Con base en esas dimensiones, se diseñaron y validaron sesenta ítems divididos en ocho factores: inmediatez/afecto (reactivo), semejanza/profundidad (reactivo), receptividad/ confianza (reactivo), serenidad (reactivo), formalidad (reactivo), dominio (reactivo), igualdad (reactivo) y orientación a la tarea (reactivo). El tipo de respuesta fue en formato tipo Likert de cinco puntos que señalan grados de acuerdo o desacuerdo. En general, esta escala se ha utilizado en las relaciones diádicas no románticas ni familiares y ha obtenido coeficientes Alfa de Cronbach de 0,42 a 0,88.

Inventario de habilidades sociales (Social Skills Inventory)

El inventario de habilidades sociales (Social Skills Inventory) de Riggio (2006) evalúa en un autorreporte ciertas destrezas básicas que subyacen a la competencia social -como las capacidades de comunicación verbal y no verbal-e identifica sus fortalezas y debilidades. El instrumento es útil en contextos como la terapia individual y de pareja, en ámbitos organizacionales y en psicología de la salud. Los individuos responden a cada pregunta en una escala tipo Likert de cinco puntos que van desde: "No me describe para nada" a "Me describe exactamente". La puntuación es global o parcial y se valoran seis habilidades: expresividad emocional, sensibilidad emocional, control emocional, expresividad social, sensibilidad social y control social.

Como se puede observar, algunas medidas abordan aspectos no verbales de comunicación en las relaciones interpersonales desde diferentes perspectivas que enriquecen las nociones de la variable, aunque el conocimiento actual de esta aún se encuentra en la fase de identificación, descripción y clasificación de signos y sistemas (Cestero, 2006), además de que el contexto sociocultural matiza o define dichos signos y sistemas (Poyatos, 2002), especialmente en el contexto romántico; además, todavía no existe una medida validada para parejas 
románticas en México. Por ello, para la presente investigación es un reto crear, con base en las medidas más representativas en el área, dos instrumentos de medición que evalúen, por un lado, la percepción de un miembro de la pareja sobre el otro en términos del lenguaje corporal y, por el otro, la interpretación en términos de lo que comunica el otro miembro de la pareja al participante que responde (ver Anexo).

\section{Método}

\section{Participantes}

Se utilizó un muestreo no probabilístico por cuota de 367 participantes ( 179 hombres y 188 mujeres) de 18 a 65 años $(M=31,91)$, de los cuales el grupo de jóvenes (de 18 a 29 años) constituyen el 59,1\%; los adultos plenos (de 30 a 45), el 23,7\%, y los adultos maduros (de 45 a 65), el 17,2\% de la muestra (Consejo Nacional de Población [CONAPo], 2012). En cuanto a la escolaridad, se tuvo la siguiente distribución: preparatoria $(37,1 \%)$ y licenciatura $(62,9 \%)$, de los cuales el 100\% tenían una pareja en el momento de responder a los instrumentos, con un tiempo en la relación desde los ocho meses hasta los 46 años $(M=9)$. De ellos, el 53,9\% son solteros, el $30 \%$ están casados y $16,1 \%$ viven en unión libre.

\section{Instrumento}

Para la investigación se diseñaron dos instrumentos para medir la variable en cuestión, así: el primero parte de la idea de operacionalizar las vías de comunicación no verbal que hacen uso principalmente de la apariencia del emisor de la información en términos de distancia corporal, contacto visual, expresiones faciales, posturas y movimiento corporal, tono de voz y contacto físico (Manusov y Patterson, 2006). Con base en esta idea, se diseñaron sesenta reactivos en formato de respuesta tipo Likert de cinco opciones de respuesta que señalan grados de acuerdo o desacuerdo.

El segundo instrumento fue diseñado para medir la comunicación no verbal desde el punto de vista relacional, es decir, la interpretación que cada miembro de la diada hace de las actitudes y conductas comunicativas del otro, dada la vinculación afectiva que existe entre ellos al formar parte de una relación amorosa (Guerrero et al., 2007). Para ello se crearon 74 reactivos en formato de respuesta tipo Likert de cinco opciones de respuesta en grados de acuerdo o desacuerdo.

\section{Procedimiento}

Los cuestionarios fueron aplicados por cuatro psicólogos que acudieron a lugares (plazas comerciales, casas, escuelas, oficinas, etcétera) donde se podían encontrar las personas que cubrieran las características de tener una pareja al momento de la aplicación y que estuvieran dispuestos a responder a las escalas en forma anónima y confidencial.

\section{Análisis de datos}

Con el fin de llegar a una medida válida y confiable, se realizaron los siguientes análisis: 1) análisis de frecuencias reactivo por reactivo, 2) aplicación de una $t$ de Student para muestras independientes (reactivo por reactivo), 3) con los reactivos que se aprobaron en los análisis anteriores, se realizó un análisis Alfa de Cronbach para evaluar la consistencia interna de la escala, y 4) se hizo el análisis de intercorrelaciones de los reactivos para determinar el tipo de rotación.

\section{Resultados}

En el caso de la "escala de comunicación no verbalpercepción" para las relaciones románticas, se encontró que 33 de los 60 ítems diseñados discriminaron ante los análisis estadísticos señalados previamente y cumplieron con los criterios establecidos por Reyes Lagunes y García y Barragán (2008). Acto seguido se analizaron los reactivos por medio de un análisis factorial exploratorio de componentes principales con rotación ortogonal (de tipo varimax) seleccionando únicamente los reactivos con cargas factoriales iguales o superiores a 0,40. El análisis factorial arrojó un Índice кмо $=0,911 \mathrm{y}$ la Prueba de Esfericidad de Bartlett $=8.496,183, g l=0,903$ y $p=0,000$, además de indicar la obtención de seis factores con valores superiores a uno que explicaron el $56,94 \%$ de la varianza. En cuanto a su coeficiente de confiabilidad Alfa de Cronbach total, se obtuvo un valor de 0,88 (ver tabla 1 ). 
Tabla 1

Análisis factorial de componentes principales para la "escala de comunicación no verbal-percepción"

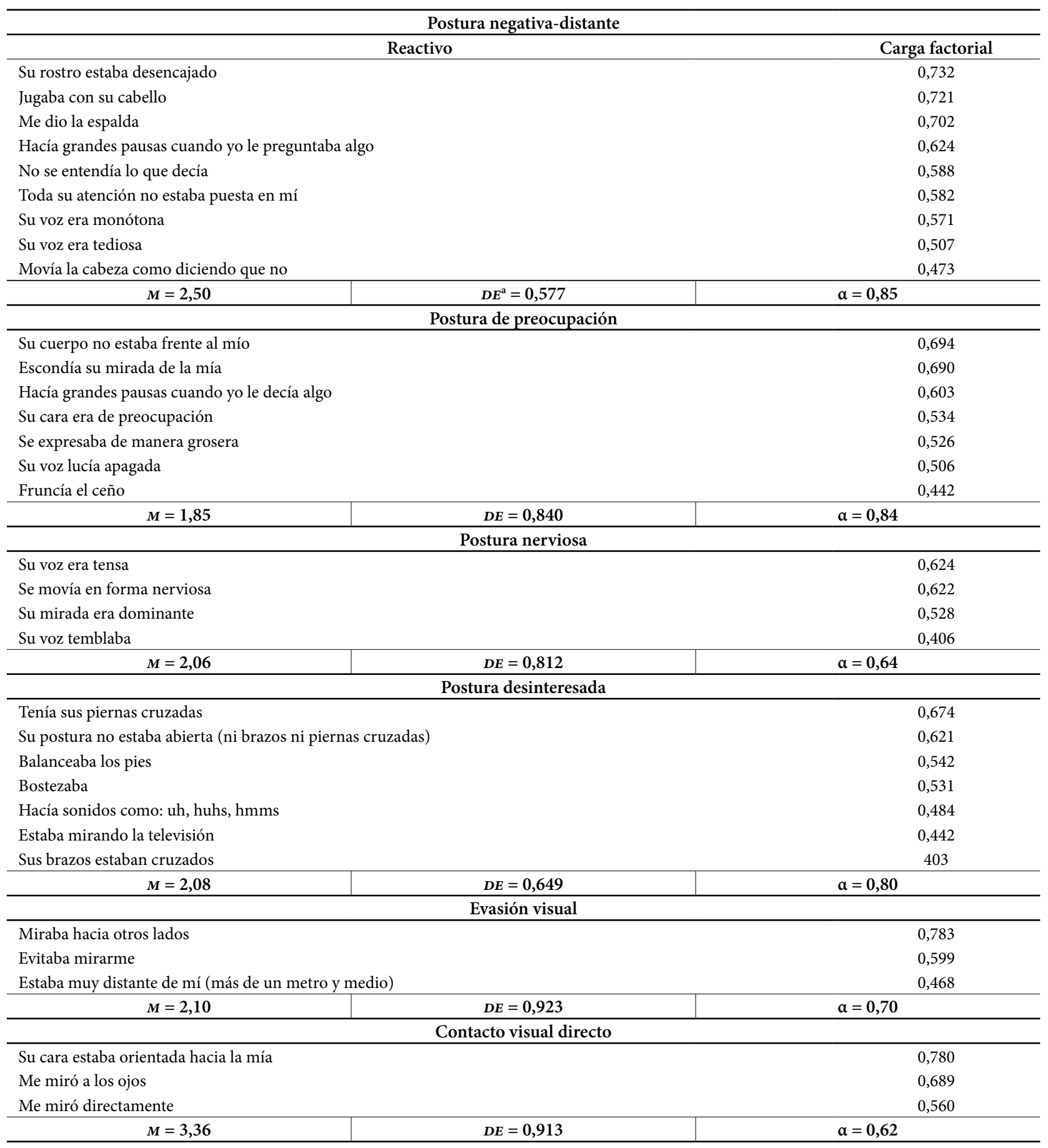

Nota. Elaboración propia. ${ }^{a} D E$ : desviación estándar

En el caso de la "escala de comunicación no verbal-interpretación" para las relaciones románticas, se realizaron los mismos análisis y con los mismos criterios que en el caso anterior, y se encontró que 39 de los 74 ítems diseñados discriminaron. En cuanto al análisis factorial exploratorio de componentes princi- pales de los reactivos, se realizó una rotación ortogonal (de tipo varimax). Dicho análisis arrojó un Índice кмо $=0,913$ y la Prueba de Esfericidad de Bartlett $=5.902,630, g l=0,666$ y $p=0,000$, además de indicar la existencia de nueve factores con valores superiores a uno que explicaron el $63,28 \%$ de la varianza 
y de los cuales únicamente cinco mostraron congruencia conceptual, no sólo con la literatura y las medidas que dieron pie a su creación, sino en su interior, ya que mostraron lógica y conveniencia (mismos que explicaron el $53,88 \%$ de varianza). Los cuatro factores elimi- nados no contaban con al menos tres reactivos para ser considerados como tales, por lo que no se consideraron apropiados para ser incluidos (Reyes-Lagunes y García y Barragán, 2008). El coeficiente de confiabilidad Alfa de Cronbach total fue de 0,92 (ver tabla 2).

Tabla 2

Análisis factorial de la escala de comunicación no verbal-interpretación para las relaciones románticas

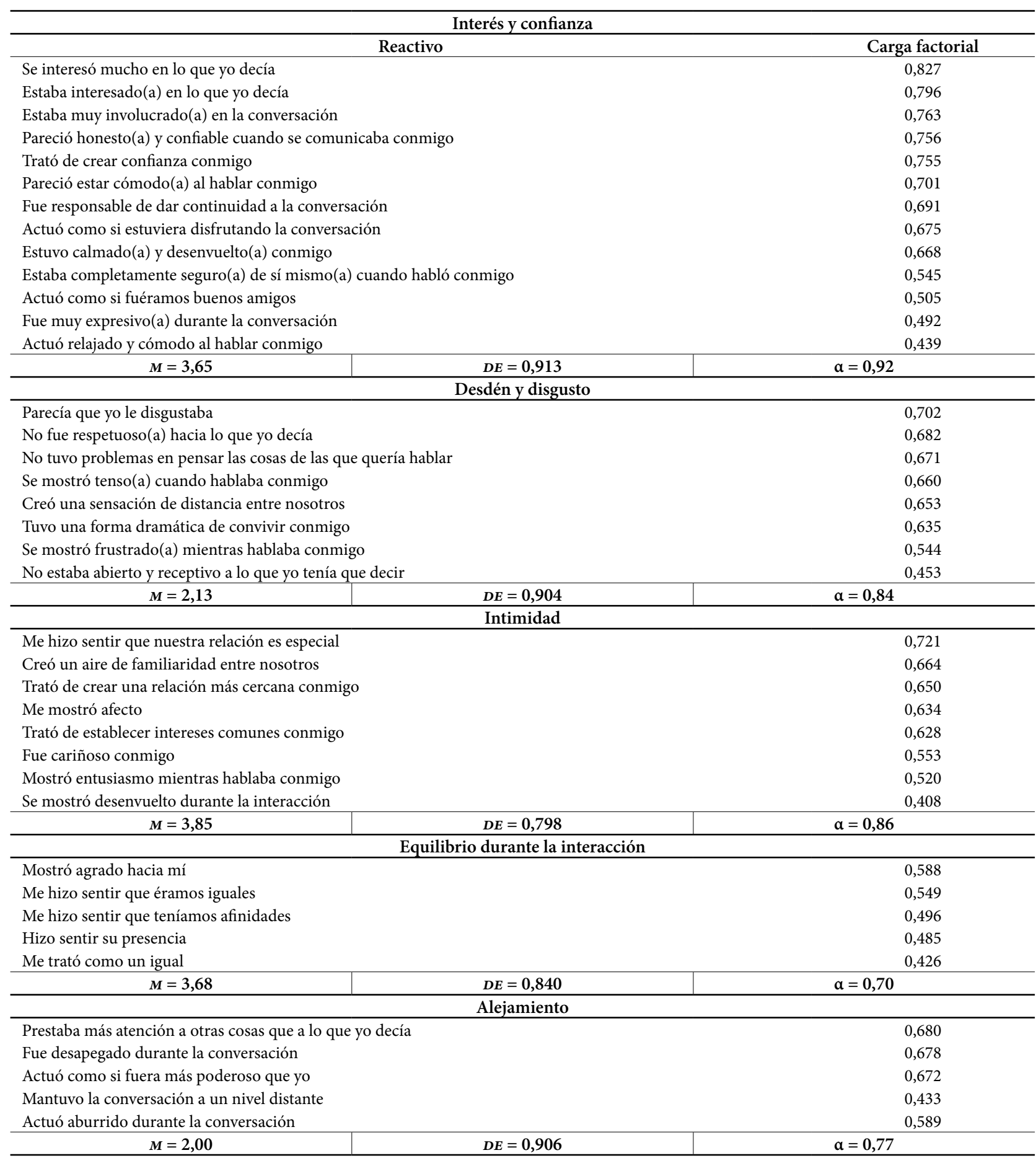

Nota. Elaboración propia. 
Como parte de la lógica de la variable en medición, se buscó explorar las relaciones entre la comuni- cación verbal en su percepción y en su interpretación. Los resultados se observan en la tabla 3.

Tabla 3

Análisis de correlación producto-momento de Pearson entre los factores de las dos medidas

\begin{tabular}{lcccccc}
\hline & $\begin{array}{c}\text { Postura } \\
\text { negativa-distante }\end{array}$ & $\begin{array}{c}\text { Postura de } \\
\text { preocupación }\end{array}$ & $\begin{array}{c}\text { Postura } \\
\text { nerviosa }\end{array}$ & $\begin{array}{c}\text { Postura } \\
\text { desinteresada }\end{array}$ & Evasión visual & $\begin{array}{c}\text { Contacto } \\
\text { visual directo }\end{array}$ \\
\hline Interés y confianza & $-0,437^{* *}$ & $-0,192^{* *}$ & $-0,124^{*}$ & $-0,251^{* *}$ & $-0,290^{* *}$ & $0,640^{* *}$ \\
Desdén y disgusto & $0,559^{* *}$ & $0,597^{* *}$ & $0,519^{* *}$ & $0,525^{* *}$ & $0,508^{* *}$ & $-0,275^{* *}$ \\
Intimidad & $-0,168^{* *}$ & $-0,500^{* *}$ & $-0,349^{* *}$ & $-0,298^{* *}$ & $-0,422^{* *}$ & $0,464^{* *}$ \\
Equilibrio durante & $-0,293^{* *}$ & $-0,279^{* *}$ & $-0,238^{* *}$ & $-0,218^{* *}$ & $-0,317^{* *}$ & $0,514^{* *}$ \\
la interacción & $0,433^{* *}$ & $0,538^{* *}$ & $0,437^{* *}$ & $0,472^{* *}$ & $0,457^{* *}$ & $-0,321^{* *}$ \\
Alejamiento & & & & & & \\
\hline
\end{tabular}

Nota. Elaboración propia.

${ }^{*} p=0,05 \quad{ }^{* *} p=0,01$

Los resultados indican que en la medida en que uno de los miembros de la pareja es percibido con una postura negativa-distante, de preocupación, nerviosa, desinteresada y de evasión visual, disminuye su transmisión de interés y confianza, de intimidad y equilibrio en la comunicación e incrementa la apreciación de evitación, disgusto y alejamiento. Por otro lado, en la medida en que se percibe que la persona usa más un contacto visual directo, tiende a comunicar más interés, confianza, intimidad y equilibrio, y menos evitación, disgusto y alejamiento.

\section{Discusión}

\section{Escala de comunicación no verbal-percepción}

En esta escala se identificaron seis factores clave que se dan durante la interacción comunicativa entre los miembros de una pareja y que se reconocen a un nivel aparente y superficial. Estos son:

- Postura negativa-distante: consta de nueve reactivos encaminados a evaluar la percepción que el participante tiene de su pareja a partir de su porte, considerando aspectos como tener el rostro desencajado, jugar con el cabello, dar la espalda, hacer grandes pausas, ser confuso al expresarse, tener una voz monótona y tediosa, no poner atención en el participante y realizar movimientos de cabeza diciendo que no. Al respecto, Mehrabian (1968 y 1969) y Burgoon, Coker y Coker (1986) señalan que estos indicadores comunican actitudes negativas, alejamiento o baja tolerancia a la intimidad mutua (véase también Burgoon, 1989; Danziger, 1982).

- Postura de preocupación: se refiere a siete expresiones que reflejan la manera como el interlocutor: no coloca su cuerpo frente a este, esconde su mirada, hace grandes pausas cuando se solicita su participación, su cara es de preocupación, se expresa en forma grosera, su voz luce apagada y frunce el ceño. Estas conductas comunican abiertamente contrariedad, ansiedad e intranquilidad (Burgoon et al., 1986).

- Evasión visual: incluye tres reactivos relativos: mirar hacia otros lados, evitar mirar al participante y posicionarse en forma distante.

- Contacto visual directo: consta de tres ítems: orientación de la cara hacia el interlocutor, mirarlo a los ojos y mirarlo directamente, cuyo papel es explicado por la literatura dentro de la interacción de la pareja. Así, para Andersen (1985), el contacto visual típicamente es crucial a la experiencia de la intimidad. Las conductas primarias oculares como mirar a otra persona o compartir miradas con ella son formas de interacción más profunda, particularmente esta última, ya que sirve como una invitación para comunicar y es vital en la generación de atribuciones de intimidad. El contacto visual es esencial para comunicar involucramiento positivo y fomentar la experiencia de intimidad en contextos cara a cara (Andersen y Andersen, 1984). Experimentos realizados por Burgoon et al. (1986) mostraron que menor contacto visual tiene efectos negativos en la intimidad percibida, comparada con niveles de contacto visual normales o mayores.

- Postura nerviosa: consta de cuatro indicadores faciales que censuran a la persona con la que se interactúa; estos son: voz tensa, movimientos nerviosos, mirada dominante y voz temblorosa. Al respecto, autores como Tucker y Anders (1998) señalan que este tipo de conductas caracterizan apropiadamente a las personas que tiene un estilo de apego ansioso. 
- Postura desinteresada: muestra siete reactivos relativos a la postura de las piernas cruzadas, representa cierta reserva o incluso posición defensiva que emerge ante una posible amenaza y que se combina con un balanceo de pies, el bostezo, las onomatopeyas o sonidos como uh, huhs y hmms, ver televisión y cruzar los brazos, que integran a esta apariencia un sentido de apatía y descuido que protege a la persona.

\section{Escala de comunicación no verbal-interpretación}

En esta escala, los factores obtenidos brindan una visión menos aparente y más evidente, al menos en el sentido y la significación del participante, al respecto de su interacción con su pareja. En torno a ello, Friedman (2001) indica que el éxito de la comunicación no verbal depende de los factores relacionados con el comunicador y con factores relativos al perceptor. En cuanto al primero, incluye las habilidades que tenga, como su motivación para ser preciso en el envío del mensaje en un contexto particular, y en cuanto al segundo, su estado emocional, su personalidad, el grado en el cual pone atención y en su motivación para entender lo que el comunicador busca transmitir.

El primer factor, "interés y confianza", plasma en trece oraciones el interés del otro en lo que se decía y en la conversación: pareció honesto, calmado, relajado, seguro e incluso que disfrutaba de la plática; creó confianza, estuvo cómodo y dio la continuidad a la conversación siendo expresivo y favoreciendo un ambiente de amistad. Sobre este tema, Andersen et al. (2006) indican que, en la expresión del amor, son clave signos como calidez, disponibilidad, cercanía y promoción del involucramiento en la interacción. Aunado a esto, Prager (2000) dice que la percepción de confianza y afecto son expresiones de intimidad que guían al enriquecimiento de las relaciones y que ayudan a manejar situaciones de conflicto en formas efectivas.

Por medio de ocho reactivos, el factor "desdén y disgusto" contempla la transmisión de molestia, falta de respeto, tensión, distanciamiento, frustración y resistencia del interlocutor ante lo que el participante deseaba comunicarle, dándole a la interacción un carácter desastroso. Con relación a esto, Vangelisti (1994) indica que dichas reacciones corresponden a los llamados mensajes hirientes y que se dan en las relaciones menos satisfactorias. Específicamente, la gente reporta más distancia y menos cercanía relacional cuando su pareja usa este tipo de mensajes. Aunque esta autora se refiere al contenido del mensaje, el factor obtenido tiene que ver con la forma en la cual -haciendo uso del lenguaje no verbal- una persona transmite su disgusto y cómo ello favorece el sufrimiento, o pasarla mal o sentirse herido en sus sentimientos.

El factor "intimidad" integra en ocho reactivos: las nociones de familiaridad, cercanía, afecto, búsqueda de afinidades, cariño, entusiasmo ante la interacción que permite fluidez en el establecimiento o fortalecimiento del vínculo. A propósito, autores como Burgoon y Hale (1984) indican que la intimidad en la comunicación incluye afecto positivo, calidez, inclusión y confianza, lo cual puede facilitar la profundidad en el vínculo.

El factor "equilibrio durante la interacción" aglomera en cinco reactivos la sensación de armonía, la complacencia, la igualdad y la afinidad durante la convivencia comunicativa, lo cual -en palabras de Devlin (2008) - se denomina calidez psicológica y favorece el mantenimiento de la relación, relajarse y sentirse cómodo. En congruencia, Argyle y Dean (1965) propusieron la teoría del equilibrio, la cual afirma que cuando las personas en interacción sienten concordia es porque buscan establecer un nivel confortable de intimidad para ambos mediante proximidad, miradas profundas y sonrisas, entre otros comportamientos.

El factor "alejamiento" consta de cinco reactivos que se refieren a la interpretación que el participante hace al respecto de una conversación con su pareja, matizada de falta de atención, desapego, dominio, distancia y aburrimiento. Al parecer, y de acuerdo con Bell, Kline y Barnard (1988), cuando una conversación es amenazante o rara, se puede generar mayor distancia de la otra persona. Por su parte, Crane, Dollahite, Griffin y Taylor (1987) indican que cuando las parejas están más insatisfechas, favorecen mayor alejamiento de su pareja ya que esto los protege de amenazas, de malos tratos o desagravios. Cabe señalar que, aunque las parejas usualmente interactúan a distancias cercanas, hay aspectos que pueden afectar esto como el momento de la relación, el tema que abordan, su estado emocional, el poder, etcétera.

\section{Referencias}

Andersen, P. A. (1999). Nonverbal Communication: Forms and Functions. Mountain View, CA: Mayfield.

Andersen, P. A. (1985). Nonverbal immediacy in interpersonal communication. En A. W. Siegaman y S. Feldstein (Eds.), Multichannel integrations of nonverbal behavior (pp. 1-36). Hillsdale, NJ: Erlbaum. 
Andersen, P. A. y Andersen, J. F. (1984). The Exchange of Nonverbal Intimacy: A Critical Review of Dyadic Models. Journal of Nonverbal Behavior, 8, 327-349.

Andersen, P. A., Guerrero, L. K. y Jones, S. M. (2006). Nonverbal Behavior in Intimate Interactions and Intimate Relationships. En V. Manusov y M. L. Patterson (Eds.). The SAGE Handbook of Nonverbal Communication (pp. 259-277). Thousand Oaks, CA: SAGE.

Argyle, M. y Dean, J. (1965). Eye Contact, Distance and Affiliation. Sociometry, 28, 289-304.

Bell, P. A., Kline, L. M. y Barnard, W. A. (1988). Friendship and Freedom of Movement as Moderators of Sex Differences in Interpersonal Distancing. Journal of Social Psychology, 128, 305-310.

Bernhard, Y. (1991). Cómo resolver conflictos de pareja. México: Pax México.

Burgoon, J. K. (1994). Nonverbal signals. En M. L. Knapp y G. R. Miller (Eds.). Handbook of Interpersonal Communication (pp. 229-285). Thousand Oaks, CA: SAGE.

Burgoon, J. K. y Hale, J. L. (1984). The Fundamental Topoi of Relational Communication. Communication Monographs, 54, 58-79.

Burgoon, J. K.; Coker, D. A. y Coker, R. A. (1986). Communicative Effects of Gaze Behavior: A Test of Two Contrasting Explanations. Human Communication Research, 12, 495-524.

Burgoon, J. K. y Hale, J. (1987). Validation and Measurement of the Fundamental Themes of Relational Communication. Communication Monographs, 54, 19-41.

Burgoon, J. K. y Baesler, E. J. (1991). Choosing Between Micro and Macro Nonverbal Measurement: Application to Selected Vocalic and Kinesic Indices. Journal of Nonverbal Behavior, 15, 57-78.

Burgoon, M. (1989). The Effects of Message Variables on Opinion and Attitude Change. En J. Bradac (Ed.), Messages in Communication Sciences: Contemporary Approaches to the Study of Effects (pp. 129-164). Newbury Park, CA: SAGE.

Cestero, A. M. (2000). El intercambio de turnos de habla en la conversación. Alcalá de Henares, Servicio de Publicaciones de la Universidad de Alcalá.

Cestero, A. M. (2006). La comunicación no verbal y el estudio de su incidencia en fenómenos discursivos como la ironía. ELUA, 20, 57-77.

Coker, D. A. y Burgoon, J. K. (1987). The Nature of Conversational Involvement and Nonverbal Encoding Patterns. Human Communication Research, 13, 463-494.

Consejo Nacional de Población (conapo). (2012). Dinámica demográfica 1990-2010 y proyecciones de población 20102030. Recuperado de: http://www.conapo.gob.mx/work/ models/conapo/Proyecciones/Cuadernos/09_Cuadernillo_DistritoFederal.pdf
Cortes, E. (2010). La auto-divulgación y la satisfacción sexual como precursores de las respuestas de insatisfacción en la pareja (Tesis inédita de Maestría). Universidad Nacional Autónoma de México, Distrito Federal, México.

Crane, D. R., Dollahite, D. C., Griffin, W. y Taylor, V. L. (1987). Diagnosing Relationships with Spatial Distance: An Empirical Test of a Clinical Principle. Journal of Marital and Family Therapy, 13, 307-310.

Danziger, K. (1982). Comunicación Interpersonal. México D. F.: El Manual Moderno.

Davidson, B., Balswick, J. y Halverson, C. (1983). Affective Self-Disclosure and Marital Adjustment: A Test of Equity Theory. Journal of Marriage and the Family, 1, 93-102.

De Paulo, B. M., Rosenthal, R., Green, C. R. y Rosenkrantz, J. (1982). Diagnosing Deceptive and Mixed Messages from Verbal and Nonverbal Cues. Journal of Experimental Social Psychology, 18, 433-446.

Deacon, T. W. (1997). The Symbolic Species: The Co-Evolution of Language and the Brain. New York: W.W. Norton.

Devlin, A. S. (2008). Judging a Book by its Cover: Medical Building Facades and Judgments of Care. Environment and Behavior, 40, 307-329.

Díaz, R. (1994). Psicología del mexicano. México: Trillas.

Estrella, R. (1991). Comunicación marital y estilos de comunicación: construcción y validación (Tesis inédita de Doctorado). Universidad Nacional Autónoma de México, Distrito Federal, México.

Floyd, K. y Burgoon, J. K. (1999). Reacting to Non-Verbal Expressions of Liking: A Test of Interaction Adaptation Theory. Communication Monographs, 66, 219-239.

Floyd, K. y Morman, M. T. (1997). Affectionate Communication in Non-Romantic Relationships: Influences of Communicator, Relational, and Contextual Factors. Western Journal of Communication, 61, 279-298.

Friedman, H. S. (2001). Paradoxes of Nonverbal Detection, Expression and Responding: Points to ponder. En J. A. Hall y F. J. Bernieri (Eds.), Interpersonal Sensitivity: Theory and Measurement (pp. 351-362). Mahwah, NJ: Erlbaum.

Gable, S., Reis, H. y Downey, G. (2003). He Said, she Said: A Quasi-Signal Detection Analysis of Daily Interactions between Close Relationship Partners. Psychological Science, 14, 100-105.

Giles, H., Coupland, J. y Coupland, N. (Eds.). (1991). Contexts of Accommodation. Cambridge, UK: Cambridge University Press.

Giles, H. y Le Poire, B. A. (2006). Introduction: The ubiquity and social meaning fullness of nonverbal communication. En V. Manusov y M. L. Patterson (Eds.), The SAGE 
Handbook of Nonverbal Communication (pp. 15-27). London: SAGE Publications.

Gonzaga, G. C., Keltner, D., Londahl, E. A. y Smith, M. D. (2001). Love and the Commitment Problem in Romantic Relations and Friendship. Journal of Personality and Social Psychology, 81, 247-262.

Gottman, J. M. y Porterfield, A. L. (1981). Communicative Competence in the Nonverbal Behavior of Married Couples. Journal of Marriage and the Family, 43, 817-824.

Guerrero, L. K. (2000). Intimacy. En D. Levinson, J. Ponzetti y P. Jorgensen (Eds.), The Encyclopedia of Human Emotions (pp. 403-409). New York: Macmillan Reference.

Guerrero, L. K. (2004). Observer Ratings of Nonverbal Involvement and Immediacy. En V. Manusonov (Ed.). The Sourcebook of Nonverbal Measures: Going beyond Words (pp. 221-235). Mahwah, NJ: Lawrence Erlbaum.

Guerrero, L. K., Andersen, P. A. y Afifi, W. A. (2007). Close Encounters: Communication in Relationships. Thousand Oaks, CA: SAGE Publications.

Hauser, M. D. (1996). The Evolution of Communication. Cambridge, MA: The mit Press.

Jones, S. E. y Yarbrough, A. E. (1985). A Naturalistic Study of the Meanings of Touch. Communication Monographs, $52,19-56$.

Kahn, M. (1970). Nonverbal Communication and Marital Satisfaction. Family Process, 9, 449-456.

Keeley, M. y Hart, A. (1994). Nonverbal Behaviors in Dyadic Interaction. En S. W. Duck (Ed.), Dynamics of Relationships (Understanding Relationships 4) (pp. 135-162). Thousand Oaks: SAGE.

Kimble, C., Hirt, E., Díaz-Loving, R., Hosch, H., Lucker, G. W. y Zárate, M. (2002). Psicología Social de las Américas. México: Prentice Hall.

Kleinke, C. L. (1986). Gaze and Eye Contact: A Research Review. Psychological Bulletin, 7, 241-246.

Knapp, M. (1997). La comunicación no verbal. Buenos Aires: Paidós Ibérica.

Levinger, G. y Senn, D. J. (1967). Disclosure of Feelings in Marriage. Merrill-Palmer Quarterly, 13, 237-249.

Manusov, V. y Patterson, M. L. (Eds.). (2006). The SAGE Handbook of Nonverbal Communication (pp. 79-95). Thousand Oaks, CA: SAGE.

Marston, P. J., Hecht, M. L., Manke, M., McDaniel, S. y Reeder, H. (1998). The Subjective Experience of Intimacy, Passion, and Commitment in Heterosexual Loving Relationships. Personal Relationships, 5, 15-30.

Mehrabian, A. (1968). Communication without Words. Psychology Today, 2, 51-52.

Mehrabian, A. (1969). Significance of Posture and Position in the Communication of Attitude and Status
Relationships. Psychological Bulletin, 71, 359-372.

Miller, S., Nunnally, E. y Wackman, D. (1976, January). A Communication Training Program for Couples. Social Casework, 57, 9-18.

Newton, D. A. y Burgoon, J. K. (1990). Nonverbal conflict behaviors: Functions, strategies, and tactics. En D. D. Cahn (Ed.), Intimates in Conflict: A Communication Perspective (pp. 77-104). Hillsdale, NJ: Lawrence Erlbaum Associates.

Noller, P. (2006). Nonverbal Communication in Close Relationships. En V. Manusonov y M. Patterson (Eds.), Handbook of Nonverbal Communication (pp. 403-420). Mahwah, NJ: Lawrence Erlbaum.

Noller, P. (2001). Using Standard Content Methodology to Assess Nonverbal Sensitivity in Dyads. En J. A. Hall y F. Bernieri (Eds.), Interpersonal Sensitivity: Theory, Measurement and Applications. Mahwah, NJ: Lawrence Erlbaum Associates.

Noller, P. (1992). Nonverbal Communication in Marriage. En R. S. Feldman (Ed.), Applications of Nonverbal Behavioral Theories and Research (pp. 31-59). Hillsdale, NJ: Lawrence Erlbaum Associates.

Noller, P. y Fitzpatrick, M. A. (1990). Marital Communication in the Eighties. Journal of Marriage and the Family, $52,832-843$.

Nowicki, S. y Duke, M. (2001). Nonverbal receptivity. En J. Hall y F. Bernieri (Eds.), Current Perspectives on Research on Nonverbal Communication (pp. 183-200). Mahwah, NJ: Erlbaum.

O’Neill, N. y O’Neill, G. (1974). Matrimonio abierto. México: Grijalbo.

Palmer, M. T. y Simmons, K. B. (1995). Communicating Intentions through Nonverbal Behaviors: Conscious and Unconscious Encoding of Liking. Human Communication Research, 22, 128-160.

Pick de Weiss, S. (1986). ¿Qué relación existe entre la percepción que se siente de la familia de origen y la satisfacción marital?. En AMEPSo (Ed.), La psicología social en México (Vol. 1, pp. 404-408). México, D. F.: Asociación Mexicana de Psicología Social.

Planalp, S. (1998). Communicating Emotion in Everyday Life: Cues, Channels, and Processes. En P. A. Andersen y L. K. Guerrero (Eds.). Handbook of Communication and Emotion: Research, Theory, Applications, and Contexts (pp. 29-48). San Diego, CA: Academic Press.

Poyatos, F. (2002). Nonverbal Communication across Disciplines: Paralanguage, Kinesics, Silence, Personal and Environmental Interaction. Amsterdam: John Benjamin Publishing Co.

Prager, K. J. (2000). Intimacy in personal relationships. En C. Hendrick y S. S. Hendrick (Eds.), Close Relationships: A Sourcebook (pp. 229-242). Thousand Oaks, CA: SAGE. 
Reyes-Lagunes, I. L. y García y Barragán, L. F. (2008). Procedimiento de validación psicométrica culturalmente relevante: un ejemplo. En S. Rivera Aragón, R. DíazLoving, R. Sánchez-Aragón e I. Reyes-Lagunes (Eds.), La psicología social en México (Vol. 7, pp. 625-636). México: Asociación Mexicana de Psicología Social.

Riggio, R. E. (2006). Nonverbal Skills and Abilities. En V. Manusov y M. L. Patterson (Eds.), The sage Handbook of Non-verbal Communication (pp. 79-95). Thousand Oaks, CA: SAGE Publications

Ruesch, J. y Bateson, G. (1951). Communication: The Social Matrix of Psychiatry. New York: Norton y Company.

Ruys, K. I. y Stapel, D. A. (2008). How to Heat Up from the Cold: Examining the Preconditions for (Unconscious) Mood Effects. Journal of Personality and Social Psycho$\log y, 94,777-791$.

Sánchez-Aragón, R. y Díaz-Loving, R. (2003). Patrones y estilos de comunicación en la pareja: diseño de un inventario. Anales de Psicología, 19(2), 257-277.

Satir, V. (1986). Relaciones humanas en el núcleo familiar. México: Pax México.

Schumm, W. R., Figley, C. R. y Jurich, A. P. (1979). Dimensionality of the Marital Communication Inventory: A Preliminary Factor Analytic Study. Psychological Reports, $45,123-128$.

Shapiro, A. y Swensen, C. H. (1977). Self-Disclosure as a Functions of Self-Concept and Sex. Journal fo Personality Assessment, 41(2), 144-149.
Shumway, S. T. y Wampler, R. S. (2002). A Behaviorally Focused Measure for Relationships: The Couple Behavior Report (cbr). The American Journal of Family Therapy, 30, 311-321.

Sundstorm, E. y Alman, I. (1976). Interpersonal Relationships and Personal Space: Research Review and Theoretical Model. Human Ecology, 4(1), 47-67.

Thayer, R. E. (1986). Activation-Deactivation Adjective Check List (ad acl): Current Overview and Structural Analysis. Psychological Reports, 58, 607-614.

Trommsdorff . G. y John, H. (1992). Decoding Affective Communication in Intimate Relationships. European Journal of Social Psychology, 22(1), 41-54.

Tucker, J. S. y Anders, S. L. (1998). Adult Attachment Style and Nonverbal Closeness in Dating Couples. Journal of Nonverbal Behavior, 22, 109-124.

Tyler, J. M. y Feldman, R. S. (2005). Deflecting Threat to One's Image: Dissembling Personal Information as a Self-Presentation Strategy. Basic and Applied Social Psychology, 27, 371-378.

Vangelisti, A. L. (1994). Messages that Hurt. En W. R. Cupach y B. H. Spitzberg (Eds.), The dark side of interpersonal communication (pp. 53-82). Hillsdale. NJ: Lawrence Erlbaum.

Watzlawick, P. (1974). An Anthology of Human Communication. Palo Alto: Science and Behaviour Books. 


\section{Anexo}

A continuación encontrará una serie de afirmaciones que describen las distintas maneras en las cuales las personas experimentan sus conversaciones con su pareja. Por favor, marque con una $\mathrm{X}$ el recuadro que refleje mejor en qué medida se da cada conducta que se menciona, o bien si está de acuerdo o no con lo que las oraciones expresan tomando en cuenta una conversación que us- ted haya tenido con su pareja, que usted recuerde y que le sea significativa.

Responda tan rápido como le sea posible, sin ser descuidado(a), utilizando la primera impresión que venga a su mente y dando sólo una respuesta a cada oración. Recuerde que sus respuestas son totalmente anónimas y confidenciales. No hay respuestas correctas ni incorrectas, sólo describen a personas como usted y como yo. Gracias por su cooperación.

\begin{tabular}{|c|c|c|c|c|c|c|}
\hline & & & \multicolumn{3}{|c|}{ Todo el tiempo } & \multirow[t]{3}{*}{5} \\
\hline & & \multicolumn{3}{|c|}{ Frecuentemente } & \multirow[t]{2}{*}{4} & \\
\hline & \multicolumn{3}{|c|}{ A veces } & \multirow[t]{3}{*}{3} & & \\
\hline & \multicolumn{2}{|c|}{ Muy poco } & \multirow[t]{2}{*}{2} & & & \\
\hline & Nunca & 1 & & & & \\
\hline \multicolumn{7}{|l|}{ MI PAREJA... } \\
\hline Su voz era tensa & & 1 & 2 & 3 & 4 & 5 \\
\hline Su cara estaba orientada hacia la mía & & 1 & 2 & 3 & 4 & 5 \\
\hline Jugaba con su cabello & & 1 & 2 & 3 & 4 & 5 \\
\hline Sus brazos estaban cruzados & & 1 & 2 & 3 & 4 & 5 \\
\hline Me miró directamente & & 1 & 2 & 3 & 4 & 5 \\
\hline Toda su atención no estaba puesta en mí & & 1 & 2 & 3 & 4 & 5 \\
\hline Su rostro estaba desencajado & & 1 & 2 & 3 & 4 & 5 \\
\hline Miraba hacia otros lados & & 1 & 2 & 3 & 4 & 5 \\
\hline Evitaba mirarme & & 1 & 2 & 3 & 4 & 5 \\
\hline Se movía en forma nerviosa & & 1 & 2 & 3 & 4 & 5 \\
\hline Movía la cabeza como diciendo que no & & 1 & 2 & 3 & 4 & 5 \\
\hline Me miró a los ojos & & 1 & 2 & 3 & 4 & 5 \\
\hline No se entendía lo que decía & & 1 & 2 & 3 & 4 & 5 \\
\hline Su postura estaba abierta (ni brazos ni piernas cruzadas) & & 1 & 2 & 3 & 4 & 5 \\
\hline Hacía grandes pausas cuando yo le preguntaba algo & & 1 & 2 & 3 & 4 & 5 \\
\hline Su voz era tediosa & & 1 & 2 & 3 & 4 & 5 \\
\hline Balanceaba los pies & & 1 & 2 & 3 & 4 & 5 \\
\hline Su voz era monótona & & 1 & 2 & 3 & 4 & 5 \\
\hline Estaba muy distante de mí (más de un metro y medio) & & 1 & 2 & 3 & 4 & 5 \\
\hline Su mirada era dominante & & 1 & 2 & 3 & 4 & 5 \\
\hline Me dio la espalda & & 1 & 2 & 3 & 4 & 5 \\
\hline Hacía sonidos como: uh, huhs, hmms & & 1 & 2 & 3 & 4 & 5 \\
\hline Bostezaba & & 1 & 2 & 3 & 4 & 5 \\
\hline Su voz lucía apagada & & 1 & 2 & 3 & 4 & 5 \\
\hline Su cuerpo no estaba frente al mío & & 1 & 2 & 3 & 4 & 5 \\
\hline Escondía su mirada de la mía & & 1 & 2 & 3 & 4 & 5 \\
\hline Hacía grandes pausas cuando yo le decía algo & & 1 & 2 & 3 & 4 & 5 \\
\hline Se expresaba en forma grosera & & 1 & 2 & 3 & 4 & 5 \\
\hline Su voz temblaba & & 1 & 2 & 3 & 4 & 5 \\
\hline Estaba mirando la televisión & & 1 & 2 & 3 & 4 & 5 \\
\hline Fruncía el ceño & & 1 & 2 & 3 & 4 & 5 \\
\hline Su cara era de preocupación & & 1 & 2 & 3 & 4 & 5 \\
\hline Hacía grandes pausas cuando yo le decía algo & & & & & & \\
\hline
\end{tabular}




\begin{tabular}{|c|c|c|c|c|c|c|}
\hline & & \multicolumn{4}{|c|}{ Totalmente de acuerdo } & \multirow[t]{2}{*}{5} \\
\hline & & \multicolumn{3}{|c|}{ De acuerdo } & \multirow[t]{2}{*}{4} & \\
\hline & \multicolumn{3}{|c|}{ Ni en desacuerdo ni en acuerdo } & \multirow[t]{3}{*}{3} & & \\
\hline & \multicolumn{2}{|c|}{ En desacuerdo } & \multirow[t]{2}{*}{2} & & & \\
\hline & Totalmente en desacuerdo & 1 & & & & \\
\hline \multicolumn{7}{|l|}{ MI PAREJA... } \\
\hline Estaba muy involucrado(a) en la conversación & & 1 & 2 & 3 & 4 & 5 \\
\hline Actuó como si fuéramos buenos amigos & & 1 & 2 & 3 & 4 & 5 \\
\hline Fue cariñoso(a) conmigo & & 1 & 2 & 3 & 4 & 5 \\
\hline Mostró entusiasmo mientras hablaba conmigo & & 1 & 2 & 3 & 4 & 5 \\
\hline Se mostró frustrado(a) mientras hablaba conmigo & & 1 & 2 & 3 & 4 & 5 \\
\hline Me hizo sentir que éramos iguales & & 1 & 2 & 3 & 4 & 5 \\
\hline Pareció honesto (a) y confiable cuando se comunicaba conmigo & & 1 & 2 & 3 & 4 & 5 \\
\hline Se mostró desenvuelto(a) durante la interacción & & 1 & 2 & 3 & 4 & 5 \\
\hline Mostró agrado hacia mí & & 1 & 2 & 3 & 4 & 5 \\
\hline Me hizo sentir que teníamos afinidades & & 1 & 2 & 3 & 4 & 5 \\
\hline Fue muy expresivo(a) durante la conversación & & 1 & 2 & 3 & 4 & 5 \\
\hline Actuaba aburrido(a) durante la conversación & & 1 & 2 & 3 & 4 & 5 \\
\hline Prestaba más atención a otras cosas que a lo que yo decía & & 1 & 2 & 3 & 4 & 5 \\
\hline Actuó como si fuera más poderoso(a) que yo & & 1 & 2 & 3 & 4 & 5 \\
\hline Fue desapegado(a) durante la conversación & & 1 & 2 & 3 & 4 & 5 \\
\hline Fue responsable de dar continuidad a la conversación & & 1 & 2 & 3 & 4 & 5 \\
\hline Creó una sensación de distancia entre nosotros & & 1 & 2 & 3 & 4 & 5 \\
\hline Parecía que yo le disgustaba & & 1 & 2 & 3 & 4 & 5 \\
\hline Se mostró tenso(a) cuando hablaba conmigo & & 1 & 2 & 3 & 4 & 5 \\
\hline No tuvo problemas en pensar las cosas de las que quería hablar & & 1 & 2 & 3 & 4 & 5 \\
\hline Se interesó mucho en lo que yo decía & & 1 & 2 & 3 & 4 & 5 \\
\hline Estaba interesado(a) en lo que yo decía & & 1 & 2 & 3 & 4 & 5 \\
\hline Hizo sentir su presencia & & 1 & 2 & 3 & 4 & 5 \\
\hline Mantuvo la conversación a un nivel distante & & 1 & 2 & 3 & 4 & 5 \\
\hline Me trató como un igual & & 1 & 2 & 3 & 4 & 5 \\
\hline Actuó como si estuviera disfrutando la conversación & & 1 & 2 & 3 & 4 & 5 \\
\hline Pareció estar cómodo(a) al hablar conmigo & & 1 & 2 & 3 & 4 & 5 \\
\hline Estaba completamente seguro(a) de sí mismo(a) cuando habló conmigo & & 1 & 2 & 3 & 4 & 5 \\
\hline Me mostró afecto & & 1 & 2 & 3 & 4 & 5 \\
\hline Trató de crear una relación más cercana conmigo & & 1 & 2 & 3 & 4 & 5 \\
\hline Trato de crear confianza conmigo & & 1 & 2 & 3 & 4 & 5 \\
\hline Creó un aire de familiaridad entre nosotros & & 1 & 2 & 3 & 4 & 5 \\
\hline Me hizo sentir que nuestra relación es especial & & 1 & 2 & 3 & 4 & 5 \\
\hline No fue respetuoso(a) hacia lo que yo decía & & 1 & 2 & 3 & 4 & 5 \\
\hline Actuó relajado (a) y cómodo (a) al hablar conmigo & & 1 & 2 & 3 & 4 & 5 \\
\hline No estaba abierto y receptivo(a) a lo que yo tenía que decir & & 1 & 2 & 3 & 4 & 5 \\
\hline Trató de establecer intereses comunes conmigo & & 1 & 2 & 3 & 4 & 5 \\
\hline Tuvo una forma dramática de convivir conmigo & & 1 & 2 & 3 & 4 & 5 \\
\hline Estuvo calmado(a) y desenvuelto(a) conmigo & & 1 & 2 & 3 & 4 & 5 \\
\hline
\end{tabular}

